\title{
System reliability modeling and life cycle research considering component characteristics and correlation
}

\author{
Xinsheng Jia ${ }^{1, \mathrm{a}}$, Youling Chen ${ }^{2, \mathrm{~b}}$ \\ ${ }^{1}$ School of Mechanical Engineering, Chongqing University, Chongqing, China \\ ${ }^{2}$ School of Mechanical Engineering, Chongqing University, Chongqing, China
}

\begin{abstract}
The accuracy and rationality of the system life depends not only on the detailed grasp of the system operation information, but also on the structural relationship between the internal components of the system and the correlation between the characteristics of each component and the degradation of the components. This article explicitly considers the different characteristics of component failure, and adopts different random process personalized modeling for the failure modes of different components; on this basis, the failure correlation between the components that work together to realize the system function is considered, and the synergistic effect of component failure is integrated into the reliability degradation model of components. In terms of system failure criteria, starting from an economic point of view, the overall working cycle of the system is obtained by comparing the relationship between the replacement cycle of lossy parts and the remaining life of the stable parts. Finally, an example is given to show the effectiveness and practicability of the proposed method.
\end{abstract}

\section{Introduction}

In modern manufacturing enterprises, in order to meet customers' demands for personalized and high-quality products, the internal equipment of enterprises is also developing towards the direction of multi-functional integration. The equipment presents the characteristics of integration, high precision and complexity, and the equipment cost is also rising due to its functions. The characteristics of high quality and just-in-time demand of products require enterprises to accurately grasp the condition of equipment, reasonably design the production plan and manage the manufacturing process[1]. In order to maximize the production capacity of equipment, accurate prediction of equipment life is the prerequisite. manufacturing enterprises, in order to respond to the customer's demand for personalized highquality products, the equipment within the enterprise is also developing in the direction of multi-functional integration. As a result, the cost of equipment has also risen. The high quality and on-time demand characteristics of products require enterprises to accurately grasp the status of equipment and reasonably design the production plan and manage the manufacturing process. In order to maximize the production capacity of the released equipment, accurate prediction of the life of the equipment is a prerequisite. Research on equipment life prediction has been carried out for decades. A large number of studies have shown that accurate life prediction can provide a basic guarantee for the orderly production and maintenance of enterprises, reduce the probability of unplanned downtime, and avoid unnecessary downtime penalties. This maximize the productivity and economic benefits of the enterprise.

Accurate prediction of equipment life cycle depends to a large extent on the consistency between the reliability degradation model of equipment components and the actual situation[2,3], and the practical reliability degradation model is the prerequisite for accurately grasping the operation status of equipment, reasonably arranging the production plan, and maximizing the production capacity of equipment. Therefore, the reliability degradation model has always been a key research content in the fields of life prediction and equipment maintenance.

In the field of reliability degradation models, there are abundant literatures on the process of reliability degradation of single-component systems. Some researchers have considered the multi-state of the degradation process in the modeling process, and divided the degradation process into normal, defect, failure and other states according to the performance characteristics of the equipment to model the reliability degradation process [4]. Some researchers have paid attention to the influence of the self-healing ability of equipment made of special materials while considering the multiple stages of the degradation process [5]. Another important research direction is to consider the degradation failure model of the system from two perspectives of system internal degradation and external shocks. Reference $[6,7]$

ajia.jxs@foxmail.com, ${ }^{\mathrm{b}}$ Youlingchencqu@163.com 
studies reliability degradation from the one-way impact of external shock on internal degradation. On this basis, they further studied the reliability degradation model when there is a the interdependency between natural degradation process and random shocks[8]. Reference [9] proposes a three-moment saddlepoint approximation approach to quantify the impact between shock and degradation.

With the development of the research, the degradation model has shifted from single-component degradation to multi-component degradation research. Some researchers do not consider the correlation between components, but only consider the heterogeneity of the degradation process of each component. In many studies on opportunistic maintenance and group maintenance, they assume that the components degenerate independently, and then select the maintenance strategy according to the degradation state of each component. Some researchers have conducted research from the perspective of the serial-parallel relationship between components (or equipment), and studied the differences in the degradation processes of various components and their impact on the quality of the final product $[10,11]$. Reference [12] considers the influence of disassembly operations on system reliability under different influencing factors, and proposes a connection matrix model that quantifies the effect of disassembly operations on component degradation processes.

Although these researches take multi-component system as the research object and only consider the series-parallel relation of components more, the degradation process of components is independent from each other, which is the motivation of the author to consider the related system reliability degradation process on the basis of the respective characteristics of components. In this paper, considering the difference of functional characteristics and working mode of components, components are divided into loss-type components and auxiliary components. Each type has its unique reliability degradation characteristics, and different random processes can be well used to show the degradation characteristics of each type of components. Due to the reality that the components cooperate to complete the system tasks, the author considers the mutual influence between the components on the basis of the degradation of the personalized reliability of the components. This effect is bidirectional and is expressed by adding a degradation impact factor in a random process. In order to make the life of the system is not simply equivalent to the life cycle of slower degrading components. This article uses an economic criterion to compare the cost and system benefits of lossy components to determine the life cycle of the system.

\section{Reliability modeling based on component correlation}

\subsection{Reliability of loss-type components}

The characteristics of the components are reflected by the differences in the working principle, degradation cycle, degradation characteristics, maintenance attributes and other elements between the components, and have relative significance. From the perspective of component characteristics, some equipment parts have consumable working characteristics (grinding wheel of grinding machine, cutting tool of lathe, etc.) relative to other parts in the equipment, with fast degradation speed, low cost of replacement, and short scrap period. This article refers to this type of component as a loss-type component.

In this paper, component reliability is selected as the characterization of component degradation status and actual operating conditions. For loss-type components, due to their relatively short replacement cycle, the abstraction of the degradation process of loss-type components is considered to follow a uniform speed degradation process during the replacement cycle, and its reliability change is also a constant speed change process, so the loss-type type The reliability of the components can be abstractly expressed as:

$$
R_{1 j}\left(t_{j}\right)=a t_{j}+1 \quad a<0
$$

$R_{1 j}(t)$ represents the reliability at the time $t_{j}$ after the jth replacement of the loss-type component, a represents the rate of decrease in reliability, and if the correlation of degradation between components is not considered, a is a constant value $\mathrm{a}_{0}$, which represents the loss-type component The rate of decline in its own reliability, in actual situations, the components always cooperate with each other to complete the expected work, so the value of a depends not only on $\mathrm{a}_{0}$, but also affected by the degradation state of the auxiliary components at time $t$, using a monotonically decreasing The function $\Psi\left(R_{2}(t)\right)$ represents the effect of the reliability of auxiliary components on the rate of decrease of the reliability of loss-type components. The final expression of a is as follows:

$$
a=a_{0}+\Psi\left(R_{2}(t)\right)
$$

Substitute (2) into (1) to obtain the reliability model of the loss-type component after the jth replacement:

$$
R_{1 j}\left(t_{j}\right)=\left(a_{0}+\Psi\left(R_{2}(t)\right)\right) t_{j}+1
$$

\subsection{Auxiliary component reliability modeling}

Compared with loss-type components, auxiliary components have completely different characteristics. Firstly, in terms of working mode, auxiliary components generally do not contact with working objects directly, but provide support, power and other auxiliary functions for loss-type components, so the working environment of auxiliary components is more stable. The relatively isolated working environment makes the degradation state of auxiliary components complex, the degradation duration is longer, and the life cycle is generally longer. The long working time provides opportunities for the realization of various maintenance methods. But the slow and complicated degradation process also leads to more economic costs once the reliability reaches the replacement threshold beyond expectations. 
In the reliability and fault analysis of equipment, exponential distribution or Weibull distribution is generally used to express the fault distribution function in the field of reliability. When modeling the reliability of auxiliary components, considering the change of the failure rate with time, it is not constant, so the Weibull distribution is chosen to describe the failure distribution of auxiliary components, then $\mathrm{f}(\mathrm{t})$ is:

$$
\mathrm{f}(t)=\frac{m}{\eta}\left(\frac{t}{\eta}\right)^{m-1} \exp \left[-\left(\frac{t}{\eta}\right)^{m}\right]
$$

$m$ is the shape parameter; $\eta$ is the scale parameter.

Then $\mathrm{F}(\mathrm{t})$ is:

$$
\mathrm{F}(t)=1-\exp \left[-\left(\frac{t}{\eta}\right)^{m}\right] \quad m_{s} \eta>0
$$

The reliability function $\mathrm{R}(\mathrm{t})$ of the component is:

$$
\mathrm{R}(t)=1-\mathrm{F}(t)=\exp \left[-\left(\frac{t}{\eta}\right)^{m}\right] \quad m_{s} \eta>0
$$

The equipment failure rate reflects the probability that a device that has not failed at a certain time will fail within a unit time after that time. Since this paper assumes that the failure condition follows Weibull distribution, its failure rate function $\lambda(t)$ is:

$$
\lambda(t)=\frac{m}{\eta}\left(\frac{t}{\eta}\right)^{m-1} \quad m, \eta>0
$$

Multi-component equipment has the characteristics of structural correlation and joint operation to complete the task, which leads to the degradation and failure of the components must be related, and this relationship is not unidirectional but bidirectional. Therefore, the replacement of loss-type component will inevitably affect the failure rate of auxiliary component. This article assumes that the change rate of the failure rate of auxiliary component is only related to the running time of auxiliary parts, but after each replacement of loss-type component will reduce the failure rate of auxiliary components by a fixed value related to the number of replacements, expressed as $\Omega(\mathrm{i})$. Then the failure rate function of the auxiliary component can be expressed as:

$$
\lambda(t)=\left\{\begin{array}{cc}
\frac{m}{\eta}\left(\frac{t}{\eta}\right)^{m-1} & t<t_{1} \\
\frac{m}{\eta}\left(\frac{t}{\eta}\right)^{m-1}-\Omega(1) & t_{1}<t<t_{2} \\
\frac{m}{\eta}\left(\frac{t}{\eta}\right)^{m-1}-\Omega(i) & t_{i}<t<t_{i+1}
\end{array}\right.
$$

From (6) and (7):

$$
\mathrm{R}(t)=\exp \left(-\int_{0}^{t} \lambda(t) d t\right)
$$

Substituting (8) into (9) can obtain the reliability model of auxiliary components under the influence of loss-type components.

\section{System life prediction of component competition failure under economic judgment criteria}

Due to the large differences in characteristics between components, there are loss-type components and auxiliary components. If the traditional equipment scrapping criteria are applied, the true system life cannot be obtained. Assuming that the reliability of the system is measured by adding the average or product of the reliability of the two components, according to the reliability obtained in this way as the criterion for system scrap, it will inevitably cause the system to be scrapped prematurely, wasting the working capacity of auxiliary components. Therefore, in this paper, considering the characteristics of short replacement cycle of loss-type components and the reliability recovery after replacement, combined with the characteristics of auxiliary components, long degradation cycle and long working life, in order to maximize the release of the working capacity of various components in the system, on the premise of satisfying the requirements of working conditions, the goal of obtaining the longest life cycle of the system is proposed, and a system life prediction model based on component characteristics of component competition failure is proposed.

The short replacement cycle of loss-type components determines the life cycle of the system. The overall reliability of the system (that is, the reliability of the system composed of two components in series or parallel) cannot be used as a judgment criterion. The end-of-life criterion, so this paper proposes a judgment criterion based on the reliability of long-period auxiliary components to meet production needs and comprehensive consideration of the economics of the update interval of loss-type components. The key problem of this economic judgment criterion is an economic comparison of the cost of replacement of losstype components and the economic benefits that the system can create during the failure threshold of the auxiliary components.

The process of calculating the life cycle of a twocomponent system containing loss-type and auxiliary components under economic criteria includes the following content. First, set the mutual influence factors $\Psi\left(R_{2}(t)\right)$ and $\Omega(\mathrm{i})$ of the two components according to the actual situation of the target system, and assume that the system starts to operate from a completely new state, that is, the reliability of both components is 1 . Then calculate the replacement interval $t_{1}$ of the loss-type component through (3), and then use (9) to calculate the reliability $R_{2}\left(t_{1}\right)$ of the auxiliary component at time $\mathrm{t}_{1}$, and substitute $R_{2}\left(t_{1}\right)$ into formula (3) to calculate The next replacement interval $t_{2}$ of the loss-type component, and finally compare the relationship between the replacement interval $t_{j+1}$ of the loss-type component and the reliability $R_{2}\left(\Sigma_{1}^{J} t_{i}\right)$ of the auxiliary type component Within the previous steps, calculate the replacement interval $t_{j+1}$ of the loss-type components and the reliability $R_{2}\left(\Sigma_{1}^{\jmath} t_{i}\right)$ of the auxiliary parts and make a judgment. If either of the two exceeds the limit or meets 
the economic judgment criteria, the life cycle of the system is output.

\section{System life prediction of component competition failure under economic judgment criteria}

According to the above, the system containing lossy and auxiliary components is abstracted into a two-component system, the loss-type component is called component 1 , the auxiliary component is called component 2 , and the mutual relationship between the two components is reasonably set according to the calculation process of the system life, the expression form of this influence factor after simulation experiment is as follows:

The influence factor of component 2 on component 1 is:

$$
\Psi\left(R_{2}(t)\right)=-\log _{1 / 35} R_{2}(t)
$$

The influence factor of component 1 on the failure rate of component 2 is:

$$
\Omega(i)=-0.0005 i+0.0025
$$

Based on the parameter setting of the reliability model in the research literature on reliability modeling and preventive maintenance strategy, reasonable deductive setting of the parameters in this paper is shown in Table 1:

TABLE I. THE VALUE OF PARAMETERS TYPE

\begin{tabular}{|c|c|c|c|c|c|}
\hline Parameters & $\mathrm{m}$ & $\eta$ & $\mathrm{a}_{0}$ & $\underline{\boldsymbol{R}}_{1}$ & $\underline{\boldsymbol{R}}_{2}$ \\
\hline Value & 3.2 & 30 & -0.03 & 0.6 & 0.6 \\
\hline
\end{tabular}

According to the system life cycle calculation process described in section 3, substituting each parameter into the corresponding formula, the replacement time interval of the loss-type component and the reliability of the component 2 at each replacement time can be calculated, as shown in Table 2 . Then, according to (3)(9)(10) and (11), the graph of the reliability change of each replacement cycle of the component 1 with time can be drawn as shown in Fig.1. At the same time, combining (8) can obtain the change graph of the failure rate of the component 2 under the influence of the replacement times of the component 1 , as shown in fig.2. The reliability change process of component 2 is further obtained, as shown in Fig.3. All graphs show the corresponding situation when there is no dependency relationship between components.

TABLE II. THE VALUE OF REPLACE INTERVAL AND RELIABILITY

\begin{tabular}{|c|c|c|c|c|}
\hline $\mathrm{j}$ & 1 & 2 & 3 & 4 \\
\hline $\mathrm{t}_{\mathrm{j}}$ & 10 & 8.27 & 4.31 & 2.72 \\
\hline$R_{2}\left(\Sigma_{1}^{\jmath} t_{\mathrm{i}}\right)$ & 0.97 & 0.83 & 0.68 & 0.57 \\
\hline
\end{tabular}

The red line in Fig.1 shows the schematic diagram of the reliability degradation of the component 1 under the influence of the component 2 . This picture shows that the reliability state of the component 2 affects the reliability degradation rate of the component 1 , and the reliability of the component 2 decreases with time (vibration Intensified, increased wear, increased temperature, etc.), leading to an increase in the rate of decrease in the reliability of the component 1 that cooperates with it to complete the work task, and the replacement cycle is shortened.

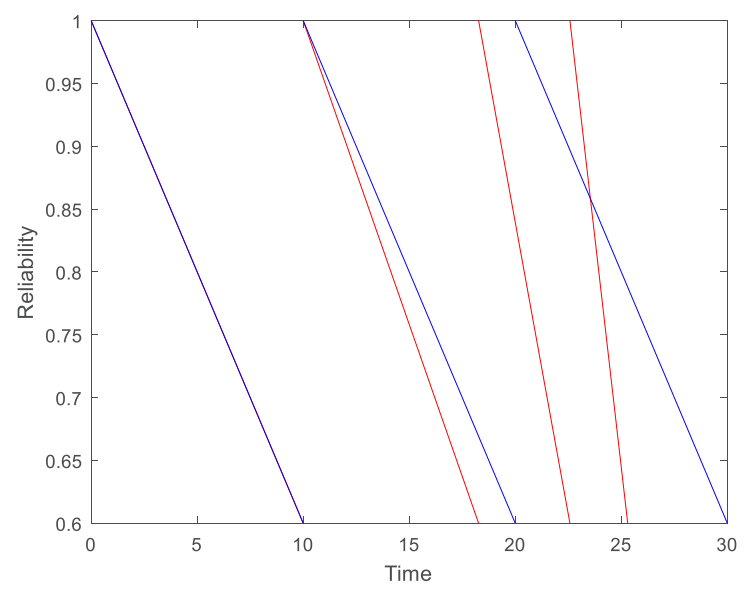

Figure 1. Component 1 reliability degradation process and replacement interval

It can be seen from the partially enlarged view in fig.2 that the failure rate of the component 2 will decrease slightly when considering the replacement operation of the component 1 compared with the independent operation, and this decline process will decay periodically with the number of replacements of the component 1 . The trend, shown in the figure, is that the interval between the two curves is getting smaller and smaller, which is consistent with the fact that the resistance of the components to faults decreases as the degree of degradation deepens.

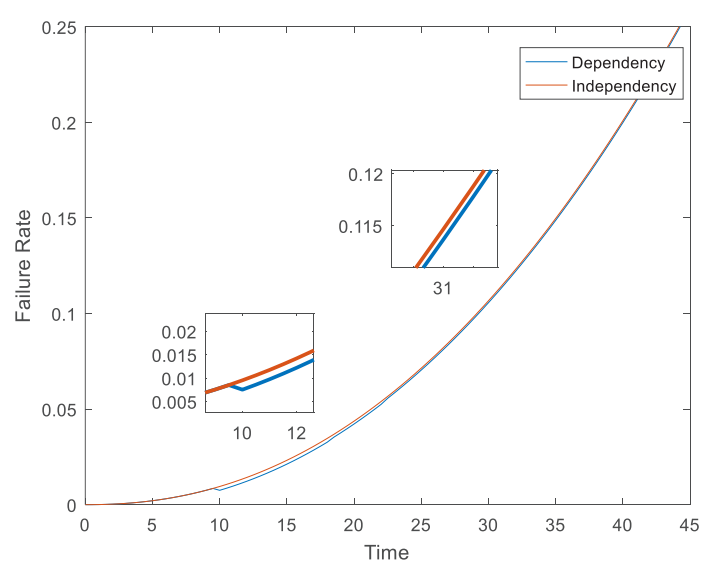

Figure 2. Comparison chart of the change of failure rate of component 2

Fig. 3 clearly explains the change in the reliability of component 2 under the influence of component 1 . It can be clearly seen that the reliability of component 2 will increase slightly with the replacement operation of component 1. However, the ability of this positive influence is gradually weakened over time, so the running time of the component 2 shows a difference under the conditions of independent degradation and mutual influence. The running time of the component 2 when considering the correlation of degradation is more than the running time of the degradation of the 
component 2 alone. In this case, the utilization rate of the device is higher.

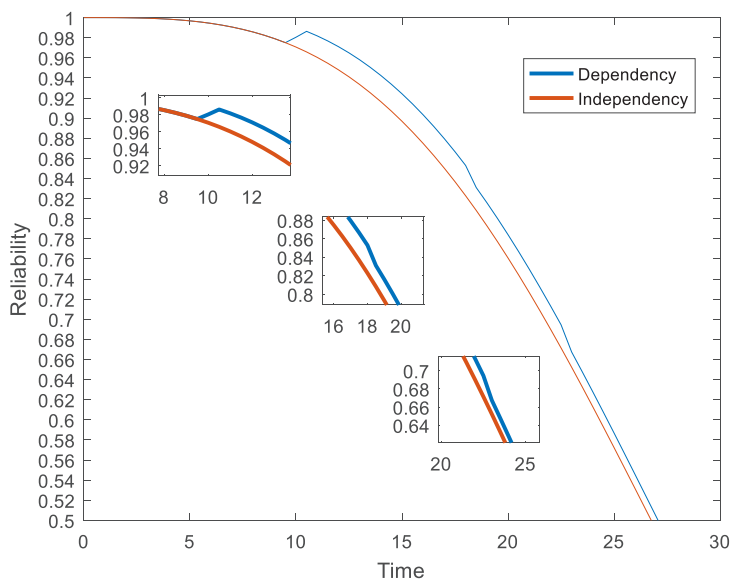

Figure 3. Component 2 reliability degradation process

In order to obtain the life cycle of the system, we must implement the economic judgment criterion. In the economic judgment criterion, if the replacement cost of component 1 is less than the economic benefit that the system can create during the failure threshold of component 2 , the time when component 2 reaches the failure threshold is used as the system life cycle, otherwise the cumulative replacement interval of component 1 is taken as the life cycle of the system. In this calculation example, combining the data in Table 2 and the drawing process in Fig.3, the life cycle of the system is 25 .

\section{Final Remark}

In this paper, two types of components (lossy type and auxiliary type) are modeled individually on the basis of considering the bidirectional influence between the component characteristics and the component reliability degradation process, and a new economic criterion is applied to determine the life cycle of the final system. The results of the numerical examples show that the two kinds of components show different characteristics from independent degradation when considering their own characteristics and mutual influence, which proves the feasibility and rationality of the proposed model and method.

The method concept mentioned in the article has practical significance, but because the author lacks practical work experience, there are some deficiencies in the research. First of all, the parameter design in this article is only derived from the existing research literature, lacking the support of a large sample database of historical data. Future researchers can obtain model parameters from rich historical data through related methods of data mining. On the other hand, the degradation model and the life cycle prediction based on the degradation model are based on the hypothesis reasoning based on the data model, and the real-time data of system degradation is not used. Therefore, in future research, the author plans to apply real-time monitoring data of the system state. Through the data fusion method, the hypothetical random model is updated in real time, thereby obtaining a more accurate life cycle.

\section{Acknowledgment}

This work was partly funded by Project supported by National key research and development plan project (No. 2018YFB1703002), and the Fundamental Research Funds for the Central Universities (No. 2018CDXYJX0019).

\section{References}

1. Y. He et al., "Reliability-oriented design of integrated model of preventive maintenance and quality control policy with time-between-events control chart," Computers \& Industrial Engineering, vol. 129, pp. 228-238, 2019/03/01/.

2. R. Kang, W. Gong, and Y. Chen, "Model-driven degradation modeling approaches: Investigation and review," Chinese Journal of Aeronautics, vol. 33, no. 4, pp. 1137-1153, 2020/04/01/

3. Z. Pang, X. Si, C. Hu, J. Zhang, and H. Pei, "A review on modeling and analysis of accelerated degradation data for reliability assessment," Microelectronics Reliability, vol. 107, p. 113602 , 2020/04/01/.

4. L. Yang, Y. Zhao, and X. Ma, "Multi-level maintenance strategy of deteriorating systems subject to two-stage inspection," Computers \& Industrial Engineering, vol. 118, pp. 160-169, 2018/04/01/.

5. X. Zhao, X. Guo, and X. Wang, "Reliability and maintenance policies for a two-stage shock model with self-healing mechanism," Reliability Engineering \& System Safety, vol. 172, pp. 185-194, 2018/04/01/.

6. J. Wang, G. Bai, Z. Li, and M. J. Zuo, "A general discrete degradation model with fatal shocks and age- and state-dependent nonfatal shocks," Reliability Engineering \& System Safety, vol. 193, p. 106648, 2020/01/01/.

7. J. Wang, Z. Li, G. Bai, and M. J. Zuo, "An improved model for dependent competing risks considering continuous degradation and random shocks," Reliability Engineering \& System Safety, vol. 193, p. 106641, 2020/01/01/.

8. J. Wang, G. Bai, and L. Zhang, "Modeling the interdependency between natural degradation process and random shocks," Computers \& Industrial Engineering, vol. 145, p. 106551, 2020/07/01/

9. X. Huang, S. Jin, X. He, and D. He, "Reliability analysis of coherent systems subject to internal failures and external shocks," Reliability Engineering \& System Safety, vol. 181, pp. 75-83, 2019/01/01/. 
10. X. Zhou and B. Lu, "Preventive maintenance scheduling for serial multi-station manufacturing systems with interaction between station reliability and product quality," Computers \& Industrial Engineering, vol. 122, pp. 283-291, 2018/08/01/.

11. A. Gouiaa-Mtibaa, S. Dellagi, Z. Achour, and W. Erray, "Integrated Maintenance-Quality policy with rework process under improved imperfect preventive maintenance," Reliability Engineering \& System Safety, vol. 173, pp. 1-11, 2018/05/01/.

12. D.-H. Dinh, P. Do, and B. Iung, "Degradation modeling and reliability assessment for a multicomponent system with structural dependence," Computers \& Industrial Engineering, vol. 144, p. 106443, 2020/06/01/. 